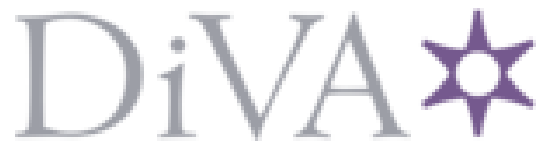

http://www.diva-portal.org

This is the published version of a paper published in BMC Cancer.

Citation for the original published paper (version of record):

Faraz, M., Tellström, A., Edwinsdotter Ardnor, C., Grankvist, K., Huminiecki, L. et al. (2020)

LRIG1 gene copy number analysis by ddPCR and correlations to clinical factors in breast cancer

BMC Cancer, 20(1): 459

https://doi.org/10.1186/s12885-020-06919-w

Access to the published version may require subscription.

N.B. When citing this work, cite the original published paper.

Permanent link to this version:

http://urn.kb.se/resolve?urn=urn:nbn:se:umu:diva-172514 


\title{
LRIG1 gene copy number analysis by ddPCR and correlations to clinical factors in breast cancer
}

\author{
Mahmood Faraz ${ }^{1}$, Andreas Tellström¹, Christina Edwinsdotter Ardnor ${ }^{1}$, Kjell Grankvist ${ }^{2}$, Lukasz Huminiecki ${ }^{3,4}$, \\ Björn Tavelin ${ }^{1}$, Roger Henriksson ${ }^{1}$, Håkan Hedman ${ }^{1}$ and Ingrid Ljuslinder ${ }^{1 *}$ (D)
}

\begin{abstract}
Background: Leucine-rich repeats and immunoglobulin-like domains 1 (LRIG1) copy number alterations and unbalanced gene recombination events have been reported to occur in breast cancer. Importantly, LRIG1 loss was recently shown to predict early and late relapse in stage I-II breast cancer.

Methods: We developed droplet digital PCR (ddPCR) assays for the determination of relative LRIG1 copy numbers and used these assays to analyze LRIG1 in twelve healthy individuals, 34 breast tumor samples previously analyzed by fluorescence in situ hybridization (FISH), and 423 breast tumor cytosols.

Results: Four of the $L R / G 1 /$ reference gene assays were found to be precise and robust, showing copy number ratios close to 1 (mean, 0.984; standard deviation, +/-0.031) among the healthy control population. The correlation between the ddPCR assays and previous FISH results was low, possibly because of the different normalization strategies used. One in 34 breast tumors (2.9\%) showed an unbalanced LRIG1 recombination event. LRIG1 copy number ratios were associated with the breast cancer subtype, steroid receptor status, ERBB2 status, tumor grade, and nodal status. Both LRIG1 loss and gain were associated with unfavorable metastasis-free survival; however, they did not remain significant prognostic factors after adjustment for common risk factors in the Cox regression analysis. Furthermore, LRIG1 loss was not significantly associated with survival in stage I and II cases.

Conclusions: Although $L R I G 1$ gene aberrations may be important determinants of breast cancer biology, and prognostic markers, the results of this study do not verify an important role for LRIG1 copy number analyses in predicting the risk of relapse in early-stage breast cancer.
\end{abstract}

Keywords: Breast cancer, LRIG1, Gene copy number, ddPCR, Prognosis

\section{Background}

Breast cancer is the most common cancer which threatens the health of women, with increasing incidence and mortality rates $[1,2]$. According to gene expression profiles, breast cancer is classified into four major subtypes: luminal A, luminal B, ERBB2-enriched (also called HER2-

\footnotetext{
* Correspondence: ingrid.ljuslinder@umu.se

'Department of Radiation Sciences, Oncology, Umeå University, SE-90187 Umeå, Sweden

Full list of author information is available at the end of the article
}

enriched), and basal-like (also called triple-negative breast cancer - TNBC) [3]. The four subtypes differ significantly with regard to incidence, response to therapy, and prognosis $[4,5]$. Even though the prognosis has improved in recent years, the risk of local recurrence remains at 10\% [6], and the distal recurrence rate is almost 30\% [7]. The most important risk factors for breast cancer outcome are tumor size, nodal involvement, tumor grade, ERBB2 status, proliferation index, and hormone receptor status [8]. However, there is a great need for new reliable factors that

C C The Author(s). 2020 Open Access This article is licensed under a Creative Commons Attribution 4.0 International License, which permits use, sharing, adaptation, distribution and reproduction in any medium or format, as long as you give appropriate credit to the original author(s) and the source, provide a link to the Creative Commons licence, and indicate if changes were made. The images or other third party material in this article are included in the article's Creative Commons licence, unless indicated otherwise in a credit line to the material. If material is not included in the article's Creative Commons licence and your intended use is not permitted by statutory regulation or exceeds the permitted use, you will need to obtain permission directly from the copyright holder. To view a copy of this licence, visit http://creativecommons.org/licenses/by/4.0/. The Creative Commons Public Domain Dedication waiver (http://creativecommons.org/publicdomain/zero/1.0/) applies to the data made available in this article, unless otherwise stated in a credit line to the data. 
can discriminate between women with a high and low risk of early and late recurrence [9].

Leucine-rich repeats and immunoglobulin-like domains protein 1 (LRIG1) is a tumor suppressor that regulates various receptor tyrosine kinases, including ERBB2 and other epidermal growth factor receptor family members [10-12]. In breast cancer, the regulation of LRIG1 expression and its impact on tumor cell fate are complex. Indeed, LRIG1 mRNA expression might be an independent prognostic marker in different subtypes of breast cancer. For example, Krig et al. found a correlation between LRIG1 mRNA expression and relapse-free survival of ER+, LN-, HER2- breast cancer patients. So in estrogen receptor (ER)-positive breast cancer, LRIG1 seems to participate in a negative feedback loop wherein estrogen signaling upregulates LRIG1 expression, which leads to the suppression of cancer cell proliferation [12]. In contrast, a feed-forward loop seems to dominate in ERBB2-positive breast cancer. Thus, whereas LRIG1 suppresses ERBB2 expression and the proliferation of ERBB2-positive breast cancer cells, ERBB2 itself downregulates LRIG1 levels in breast cancer cells, thereby canceling the tumor-suppressive function of LRIG1 [13]. Additionally, LRIG1 seems to play an important role in basal-like breast cancer. LRIG1 suppresses epithelial-tomesenchymal transition and invasion of basal-like breast cancer cells; however, LRIG1 is downregulated by unknown mechanisms in the majority of basal-like tumors [14]. Thus, LRIG1 may be an influential determinant of all the major subtypes of breast cancer, including ERpositive, ERBB2-positive, and basal-like breast tumors.

LRIG1 expression is often downregulated in cancer cells, and high expression is associated with improved survival in many cancer types (reviewed in [15]). In ERpositive and lymph node-negative breast cancer, LRIG1 mRNA expression is correlated with prolonged relapsefree survival [12], and in a series of mixed breast cancer cohorts, low expression of LRIG1 was correlated with a shorter distant metastasis-free survival (MFS) and overall survival (OS) [16]. The LRIG1 gene has shown both increased and decreased copy numbers in breast cancer. In our previous studies, in which fluorescence in situ hybridization (FISH) was used to determine gene copy numbers, LRIG1 showed increased and decreased copy numbers in 34 and $3.5 \%$ of breast tumors, respectively $[17,18]$. However, in a more recent study of stage I-II patients, which utilized a molecular inversion probe analysis platform, only $3.9 \%$ of the breast cancers showed an increased LRIG1 copy number, whereas $8.9 \%$ showed losses [16]. The same study also indicated a common breakpoint in LRIG1; however, the frequency of this event was not determined. Thus, the frequencies of LRIG1 gains, losses, and breaks in breast cancer remain controversial. Intriguingly, the study by Thompson et al.
[16] has demonstrated that LRIG1 loss predicts both early and late relapse in early-stage breast cancer. This finding is of potentially urgent clinical importance because markers for risk of late relapse in early-stage breast cancer are urgently needed.

We undertook the current study to establish a simple, precise, and sensitive droplet digital polymerase chain reaction (ddPCR) assay for the quantification of LRIG1 gene copy numbers in cells and tissues, and we applied this assay to investigate the frequency of unbalanced LRIG1 gene recombination events in breast cancer, determine the frequency of LRIG1 gains and losses in a well-characterized breast cancer cohort, and validate, or refute, the previous claim that LRIG1 loss can predict early and late relapses in breast cancer. We also performed exploratory analyses and investigated other possible associations between LRIG1 copy numbers and various clinical parameters of interest.

\section{Methods \\ Droplet digital PCR}

Primers and probes for ddPCR for the reference genes (Table S1) and different genomic positions of LRIG1 (Table S2) were purchased from Integrated DNA Technologies (Leuven, Belgium). For ERBB2, a ready-to-use ddPCR copy number variation assay was purchased from Bio-Rad Laboratories AB (Solna, Sweden; cat $\neq 10,031$, 240). The final concentrations of forward and reverse primers were $400 \mathrm{nM}$ for LRIG1 and the reference genes and $900 \mathrm{nM}$ for ERBB2. The final concentrations of the probes were $200 \mathrm{nM}$ for LRIG1 and the reference genes and $250 \mathrm{nM}$ for ERBB2. ddPCR supermix (no dUTP) (Bio-Rad, cat $\neq 1,863,024$ ), Hind III restriction enzyme (Thermo Scientific, FastDigest, cat $\neq$ FD0505), and nuclease-free water were mixed with primer/probe sets of LRIG1 or ERBB2 and primer/probe sets for the reference gene. Droplets were generated using a QX200 droplet generator followed by PCR using a T100 thermal cycler (Bio-Rad) with PCR parameters of $37^{\circ} \mathrm{C}$ for $5 \mathrm{~min}$; $95^{\circ} \mathrm{C}$ for $5 \mathrm{~min} ; 40$ cycles of $30 \mathrm{~s}$ at $95^{\circ} \mathrm{C}$ and $1 \mathrm{~min}$ at $58^{\circ} \mathrm{C}$; followed by $98^{\circ} \mathrm{C}$ for $10 \mathrm{~min}$. After PCR amplification, to acquire these data, the plate was loaded into the QX200 droplet reader (Bio-Rad). The data were analyzed using QuantaSoft software (Bio-Rad, version 1.7.4.0917). To provide good quality and consistent data, the amplitude thresholds were set to 3500 for LRIG1 or ERBB2 and 3000 for CYP1B1 in the 1-D and 2-D plots. If the total number of events was less than 8000 counts, they were not included in the final analysis. In addition, data with a coefficient of variation (CV) greater than $10 \%$ in technical replicates were removed to obtain a more precise estimation of the ratios. Researchers were blinded to the clinical data of the patients at the time of performing the ddPCR and data analysis. 


\section{Patients and tumor samples}

The breast cancer cohort analyzed in the current study comprised 423 unselected women from the Northern Region in Sweden diagnosed with primary invasive breast carcinoma between 1987 and 1999. We used the frozen cytosol samples which were prepared for steroid receptor analysis as previously described [19]. We did not purify the DNA from samples because preliminary experiments showed that the crude cytosols worked as efficiently as the purified DNA as templates in the ddPCR assays. The receptor concentration was expressed in femtomoles of receptor per $\mu \mathrm{g}$ of DNA, and tumors with a value lower than 0.1 fmol ER or progesterone receptor (PR) per $\mu$ g of DNA were considered to be receptor-negative; those with a value $\geq 0.1$ fmol ER or PR per $\mu \mathrm{g}$ of DNA were considered to be receptor-positive [20]. The International Union Against Cancer guidelines (UICC-TNM) for tumor classification and staging were used. Details of the characteristics of the patients are presented in Table 1. Primary treatment was administered according to the guidelines of the North Swedish Breast Cancer Group. Patients with node-negative disease had a modified radical mastectomy or sector resection, and the patients who underwent sector resection were treated with postoperative radiation therapy. Moreover, 60 patients received adjuvant chemotherapy, and 145 patients received adjuvant endocrine treatment, with most cases receiving tamoxifen daily for 2 to 5 years. Patients with nodepositive disease were treated with modified radical mastectomy, axillary dissection, and postoperative radiation therapy. The number of patients for whom data were available varied among the different prognostic factors studied depending on the clinical routines at the time of collection of the respective sample. Information on the histopathologic grade was available in 363 cases. The median age at diagnosis was 60 years. The last follow-up dates for OS and MFS were June 30, 2017 and December 31, 2013, respectively. Clinical information including primary stage, adjuvant therapy, time and type of relapses, and survival, was obtained from national registries and from patient records when available. Because the cohort was more than 25 years old, reliable treatment data for patients with primary metastasized disease and/or recurrence could not be obtained. The patients who were diagnosed with stage IV disease less than 6 months after their original breast cancer diagnosis were classified as primary stage IV cases in our analysis of recurrence risk. Among the 154 patients who had died from breast cancer at the last follow-up date, the date of recurrence was obtained in 96 of 154 patients and was used when analyzing time to recurrence. MFS and OS were calculated as the time from diagnosis to the date of first recurrence or death. The follow-up times for patients without documented recurrences or death were calculated as the time from diagnosis until the last clinical examination (last follow-up date, December 31, 2013).

\section{Statistical analyses}

All statistical analyses were performed using SPSS software, version 24 (IBM Corporation, Armonk, NY, USA). The Spearman correlation coefficient was used to evaluate the correlation between the LRIG1 copy number and $E R B B 2$ copy number ratios. The Kruskal-Wallis test was conducted to evaluate whether the distribution of LRIG1 copy number ratios was the same among different subtypes. Fisher's exact test (2-sided) was used to investigate the relationships between LRIG1 loss or gain with all other variables used in the cohort (Table 1). The survival analysis data were presented with Kaplan-Meier survival curves and evaluated by the log-rank (Mantel-cox) test. Cox regression analysis was also conducted for both OS and MFS, including LRIG1 loss or gain together with other prognostic factors. In all statistical analyses, the significance level was set to 0.05 .

\section{Results \\ Identification of reference genes and design and validation of ddPCR assays}

As candidate genomic reference loci, we chose six loci with a low copy number variance in breast cancer. Thus, we excluded chromosome arms and regions that were previously shown to display frequent copy number alterations in early-stage breast cancer [21], i.e., chromosomes 1q, 8, 11, 16, 17, and 20, as well as all other regions that showed gains or losses in $\geq 10 \%$ of any of the major breast cancer subtypes. Among the remaining chromosomal regions, we attempted to manually identify one or more genes per chromosome arm. However, we failed to identify suitable genes in the low-variance parts of chromosomes 4, 5, 6, 10p, 12p, 13p, 14, 15p, 18p, 21p, 22 , or Xp. In total, 23 genes on 17 different chromosome arms were identified and chosen for further evaluation (Table S3). The copy number variance among these 23 genes was analyzed in the cancer genome atlas (TCGA) breast cancer data set, revealing a frequency of copy number changes in the TCGA cohort between 0.94 and 4.1\% (Table S3). LRIG2 was excluded as a reference gene in the present study due to an apparent risk that its copy number might not be independent of the studied gene, LRIG1. Thereafter, ddPCR assays for the six reference genes that showed the lowest frequency of copy number variation in the TCGA data set and, simultaneously, were located on different chromosomal arms, were designed (Table S1). Additionally, ddPCR assays for six loci along the LRIG1 gene were designed (Table S2). The performance of all twelve ddPCR assays was good, with PCR amplification efficiencies $>94 \%$ (95\% confidence intervals [CIs] for all assays were within $0.93<1.02$ ) and good linearity $\left(\mathrm{r}^{2}=1.00\right.$ for all assays $)$ when synthetic DNA was used as the PCR template. Next, six different $L R I G 1 /$ reference gene duplex assays were used to 
Table $1 \angle R / G 1$ copy number ratios and clinicopathological characteristics of the breast cancer cohort

\begin{tabular}{|c|c|c|c|c|}
\hline Characteristic & $\operatorname{Loss}^{\mathbf{a}}$ & Normal $^{\mathbf{a}}$ & Gain $^{\mathbf{a}}$ & $\boldsymbol{P}$-value* \\
\hline Number of patients $(N=423$ & $77(18.2 \%)$ & $293(69.3 \%)$ & $53(12.5 \%)$ & \\
\hline Age at diagnosis (years; mean \pm SD) & $55.7 \pm 13.3$ & $60.1 \pm 11.54$ & $62.5 \pm 13.42$ & \\
\hline Age $\leq 60(N=211)$ & $46(21.8 \%)$ & $142(67.3 \%)$ & $23(10.9 \%)$ & 0.125 \\
\hline Age $>60(N=212)$ & $31(14.6 \%)$ & $151(71.2 \%)$ & $30(14.2 \%)$ & \\
\hline Steroid receptor status & & & & $<0.001$ \\
\hline Negative $(N=117)$ & 39 (33.3\%) & $65(55.6 \%)$ & $13(11.1 \%)$ & \\
\hline Positive $(N=306)$ & $38(12.4 \%)$ & $228(74.5 \%)$ & $40(13.1 \%)$ & \\
\hline ERBB2 status & & & & 0.002 \\
\hline Negative $(N=336)$ & $50(14.9 \%)$ & $245(72.9 \%)$ & $41(12.2 \%)$ & \\
\hline Positive $(N=87)$ & $27(31.0 \%)$ & $48(55.2 \%)$ & $12(13.8 \%)$ & \\
\hline Tumor subtype & & & & $<0.001$ \\
\hline$E R B B 2+, E R / P R-(N=45)$ & $16(35.6 \%)$ & $23(51.1 \%)$ & $6(13.3 \%)$ & \\
\hline$E R B B 2+, E R / P R+(N=42)$ & $11(26.2 \%)$ & $25(59.5 \%)$ & $6(14.3 \%)$ & \\
\hline$E R B B 2-, E R / P R+(N=264)$ & $27(10.2 \%)$ & $203(76.9 \%)$ & $34(12.9 \%)$ & \\
\hline ERBB2-, ER/PR- $(N=72)$ & $23(31.9 \%)$ & $42(58.3 \%)$ & $7(9.7 \%)$ & \\
\hline Disease stage $(N=278 ;$ missing data $=145)$ & & & & 0.151 \\
\hline I $(N=109)$ & $18(16.5 \%)$ & $84(77.1 \%)$ & $7(6.4 \%)$ & \\
\hline$\|(N=131)$ & $28(21.4 \%)$ & $85(64.9 \%)$ & $18(13.7 \%)$ & \\
\hline$\| I I(N=7)$ & $1(14.3 \%)$ & $5(71.4 \%)$ & $1(14.3 \%)$ & \\
\hline $\mathrm{IV}(N=31)$ & $6(19.4 \%)$ & $17(54.8 \%)$ & $8(25.8 \%)$ & \\
\hline Tumor grade $(N=363$, missing data $=60)$ & & & & 0.004 \\
\hline I $(N=31)$ & $1(3.2 \%)$ & $25(80.6 \%)$ & $5(16.1 \%)$ & \\
\hline$\|(N=137)$ & $15(10.9 \%)$ & $104(75.9 \%)$ & $18(13.1 \%)$ & \\
\hline III $(N=195)$ & $50(25.6 \%)$ & $120(61.5 \%)$ & $25(12.8 \%)$ & \\
\hline Tumor size $(N=329$, missing data $=94)$ & & & & 0.158 \\
\hline Size $\leq 20 \mathrm{~mm}(N=154)$ & $29(18.8 \%)$ & $111(72.1 \%)$ & $14(9.1 \%)$ & \\
\hline Size $>20 \mathrm{~mm}(N=175)$ & $37(21.1 \%)$ & $111(63.4 \%)$ & $27(15.4 \%)$ & \\
\hline Nodal status $(N=360$, missing data $=63)$ & & & & 0.001 \\
\hline Negative $(N=279)$ & $43(15.4 \%)$ & $207(74.2 \%)$ & $29(10.4 \%)$ & \\
\hline Positive $(N=81)$ & $24(29.6 \%)$ & 43 (53.1\%) & 14 (17.3\%) & \\
\hline Tumor types & & & & 0.041 \\
\hline Ductal $(N=354)$ & 66 (18.6\%) & 237 (66.9\%) & $51(14.4 \%)$ & \\
\hline Lobular $(\mathrm{N}=35)$ & 5 (14.3\%) & $30(85.7 \%)$ & $0(0.0 \%)$ & \\
\hline Others $(N=34)$ & $6(17.6 \%)$ & $26(76.5 \%)$ & $2(5.9 \%)$ & \\
\hline
\end{tabular}

a LRIG1/CYP1B1 ratio $<0.85$, loss; 0.85-1.15, normal; $>1.15$, gain

*The overall $P$ values are from comparisons between all $L R I G 1$ loss, $L R I G 1$ normal and $L R I G 1$ gain groups. Significance was calculated by the 2-sided Fisher's exact test

analyze the chromosomal DNA from twelve healthy individuals (Table S4). Four of the six assay pairs, i.e., LRIG1-9/GJB2, LRIG1-11/CHUK, LRIG1-7/CYP1B1, and LRIG1-12/NR5A1, showed ratios that were very close to 1 in all samples (mean ratios, \pm standard deviations [SD]: 0.997, $\pm 0.050 ; 0.991, \pm 0.029 ; 0,979, \pm 0.041$; and $0.968, \pm 0.030$, respectively). When these four assays were combined and used to determine the LRIG1 copy number among the twelve healthy individuals, the apparent mean copy number ratios were, on average, 0.984 (SD, \pm 0.031; 95\% CI, 0.966-1.002).

\section{LRIG1 and ERBB2 copy number variations in breast cancer tumors}

The four LRIG1/reference gene ddPCR assay pairs that had shown the ratios closest to 1 among the samples from the healthy individuals were thereafter used to analyze DNA from 34 breast cancer tumors that had 
been analyzed for LRIG1 copy number variations by FISH in a previous study [18]. The major clinical characteristics of these patients are presented in Table S5. To detect unbalanced gene recombination events, we analyzed the SD among the ratios for the four assays that were distributed along the LRIG1 gene. One sample showed an aberrant SD that was greater than 0.1 (SD, \pm 0.431 ), thus representing a probable unbalanced gene recombination event. Based on this finding, we concluded that $2.9 \%(1 / 34)$ of the breast tumors in this series had undergone an unbalanced LRIG1 gene recombination event. We used the same cut-offs as were used by us in the paper by Thompson et al., (2014); that is, the definition of loss was an LRIG1-ratio $<0.85$ and of gain a ratio $>1.15$, that is delta $+/-0.15$ around 1.00 . Using these thresholds, $11.8 \%(4 / 34)$ of the tumors showed LRIG1 loss and 2.9\% (1/34) showed LRIG1 gain. Intriguingly, only one in seven tumors that had previously shown LRIG1 gain by FISH also showed an LRIG1 gain by the
ddPCR assay. In fact, there was a poor correlation between the LRIG1 copy number ratios determined by ddPCR and the LRIG1 copy numbers previously determined by FISH (linear regression, $\mathrm{y}=1.004+0.100 \mathrm{x}$, $\mathrm{r}^{2}=0.009$; Fig. S1). Finally, we analyzed the LRIG1/ $C Y P 1 B 1$ ratio and $E R B B 2 / C Y P 1 B 1$ ratio in 423 breast cancer tumor cytosols. Here, only a single reference gene, $C Y P 1 B 1$, was used, to reduce the number of ddPCR runs. Figure 1a and $\mathrm{b}$ show the distribution of LRIG1/ $C Y P 1 B 1$ and ERBB2/CYP1B1 copy number ratios, respectively, among the 423 tumors. Using cut-offs $<0.85$ for LRIG1 loss and > 1.15 for LRIG1 gain, $18.2 \%$ of the tumors showed loss and $12.5 \%$ showed gain (Table 1). The samples with $E R B B 2 / C Y P 1 B 1$ ratios $\geq 2$ were defined as $E R B B 2$-positive tumors (according to the guideline recommendations of the American Society of Clinical Oncology/ College of American Pathologists), which corresponded to $20.6 \%$ of all tumors. Using continuous data, LRIG1 and $E R B B 2$ copy number ratios were correlated $(P=0.016$,

\section{A}

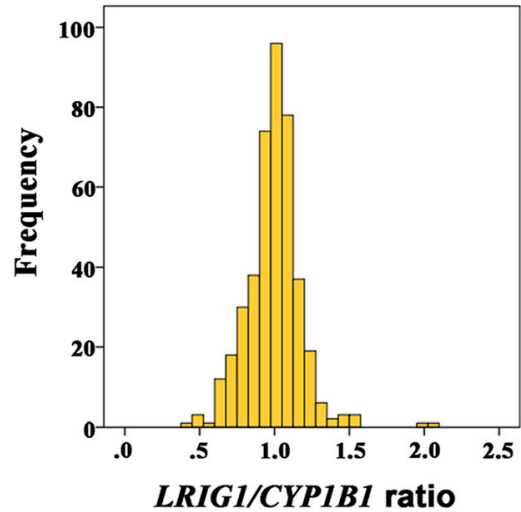

C

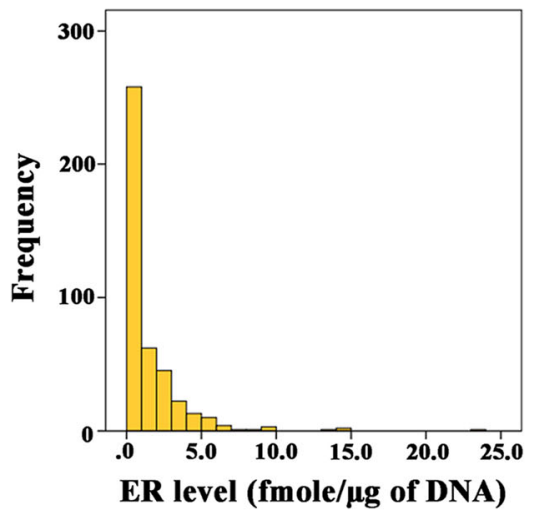

B

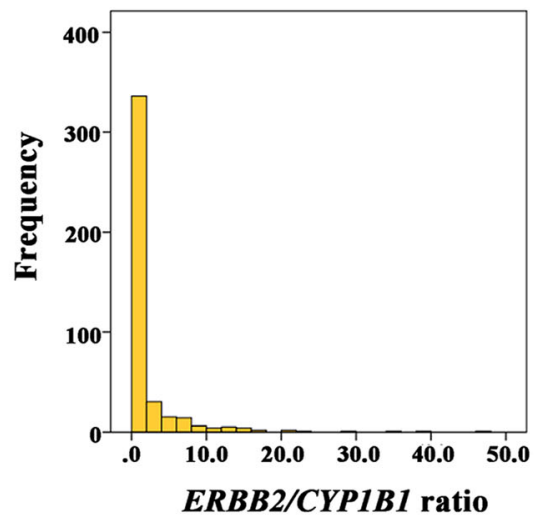

D

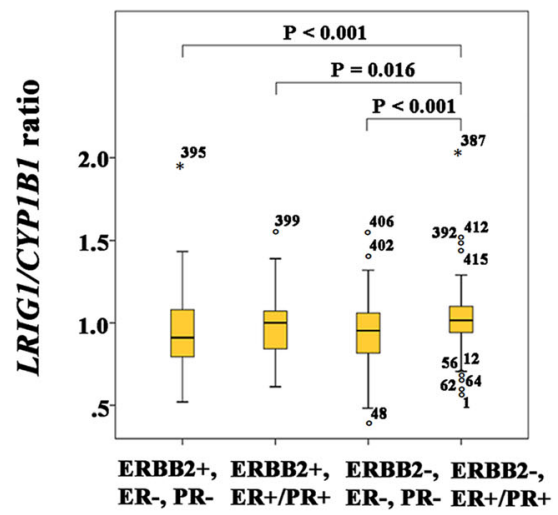

Fig. 1 Frequency distributions of $L R I G 1$ and ERBB2 copy number ratios and ER levels and relationships between $L R I G 1$ copy number ratios and breast cancer subtypes among 423 breast cancer cases. a Frequency distributions of $L R / G 1 / C Y P 1 B 1$ ratios determined by ddPCR. b Frequency distributions of ERBB2/CYP1B1 ratios determined by ddPCR (c) Frequency distributions of ER levels retrieved from clinical records. $\mathbf{d}$ Box plots of LRIG1/CYP1B1 ratios for each tumor subtype 
Spearman's $\rho$ correlation coefficient $=0.117$ ). Nevertheless, LRIG1 loss was more common among the ERBB2positive (31\%) than among the ERBB2-negative (14.9\%) tumors $(P=0.001$, Fisher's exact test, 2 -sided). The frequency of LRIG1 gains did not differ between the ERBB2positive and ERBB2-negative tumors $(P=0.323$, Fisher's exact test, 2-sided).

We also investigated the effects of minor changes of the cut-off levels. New cut-offs were tested with delta from 0.15 up to 0.25 with step 0.01 . When these alternative cut-off definitions were tested in the full model, together with the other prognostic factors, each level of LRIG-ratio was found to be non-significant. This means that the definition of loss and gain used in the manuscript was stable and not dependent on minor changes in the predefined cut-offs.

\section{Associations between LRIG1 losses or gains and various clinical parameters}

Figure 1c shows the distribution of ER levels in the cohort. The median and mean values of ER were $0.6 \mathrm{fmol} / \mu \mathrm{g}$ of DNA and $1.4 \mathrm{fmol} / \mu \mathrm{g}$ of DNA, respectively (range from 0.0 to $23.0 \mathrm{fmol} / \mu \mathrm{g}$ of DNA). The median and mean values of PR were $0.4 \mathrm{fmol} / \mu \mathrm{g}$ of DNA and $1.4 \mathrm{fmol} / \mu \mathrm{g}$ of DNA, respectively (range from 0.0 to $22.0 \mathrm{fmol} / \mu \mathrm{g}$ of DNA). LRIG1 loss was more common among steroid receptor-negative (33.3\%) than among steroid receptor-positive (12.4\%) tumors $(P<0.001$, Fisher's exact test, 2 -sided) (Table 1$)$. The frequency of LRIG1 gain did not differ between steroid receptor-negative and steroid receptor-positive tumors ( $P=0.722$, Fisher's exact test, 2-sided). We defined four breast cancer subtypes in our study based on the data for $E R B B 2$ copy numbers and ER and PR receptor statuses: $E R B B 2+$, ER/PR- (i.e., ERBB2+, ER-, PR-); $E R B B 2+$, ER/ $\mathrm{PR}+$ (i.e., $E R B B 2+, \mathrm{ER}+, \mathrm{PR}-; E R B B 2+, \mathrm{ER}-, \mathrm{PR}+$; or $E R B B 2+$, ER+, $\mathrm{PR}+$ ); ERBB2-, ER/PR+ (i.e., ERBB2-, ER+, PR-; ERBB2-, ER-, PR+; or ERBB2-, ER+, $\mathrm{PR}+$ ); and $E R B B 2-$, ER/PR- (i.e., ERBB2-, ER-, PR-). Figure 1d shows the LRIG1 copy number ratios among the breast cancer subtypes. LRIG1 copy number ratios were different among the groups $(P<0.001$, Kruskal-Wallis test). In a pairwise comparison, LRIG1 loss was less common among the $E R B B 2-, E R / P R+$ tumors than the other subtypes $(P=$ 0.016 , Fisher's exact test, 2 -sided). We defined disease stage from I to IV based on the TNM staging system. The TNM data for 145 patients were missing. There were only seven stage III patients, among whom only one patient had a loss and another had a gain. The frequencies of LRIG1 loss did not differ among various disease stages (Fisher's exact test); however, LRIG1 gain was more common in stage IV than in stage I $(P=0.004$, Fisher's exact test, 2 -sided). Tumor grade data were available for 363 patients. Among those tumors, LRIG1 loss was more common among grade 3 tumors than among grade 1 tumors and was more common among grade 3 tumors than among grade 2 tumors; however, there was no difference between grade 1 and grade 2 tumors $(P=0.004, P=0.001$, and $P=0.305$, respectively, Fisher's exact test, 2-sided). LRIG1 gain was equally common among the different tumor grades (Fisher's exact test). LRIG1 copy number ratios were not correlated with tumor size. Both LRIG1 loss and gain were significantly correlated with nodal status $(P=0.002$, and $P=0.035$, respectively, Fisher's exact test, 2-sided). Node-positive tumors had more LRIG1 losses or gains than node-negative tumors. The frequencies of LRIG1 losses differed among ductal, lobular, and "others" tumor types $(P=0.041$, Fisher's exact test). Among the tumors with lobular cancer, no LRIG1 gain was found $(0 / 35)$.

\section{Patient survival analyses}

First, we confirmed the associations between known prognostic factors and patient MFS in our cohort by applying the Mantel-Cox log-rank tests (Fig. S2). Steroid receptor-negative patients had a worse MFS than steroid receptor-positive patients $(P<0.001$, Fig. S2A). ERBB2amplification was strongly correlated with a worse MFS $(P<0.001$, Fig. S2B). Among our four defined breast cancer subtypes, the ERBB2-, ER/PR+ subtype showed the best MFS, whereas the ERBB2+, ER/PR- subtype had the worst prognosis (Fig. S2C). There were significant differences in MFS between the ERBB2-, ER/PR+ subtype and all other subtypes $(P=0.002)$ and between the $E R B B 2+$, ER/PR- and ERBB2-, ER/PR- subtypes $(P=0.048)$ $(P<0.001)$. Tumor grade stratified patients into three different prognostic groups, among which patients with a higher grade had a worse MFS $(P=0.014$, Fig. S2D). Similarly, tumor size stratified the patients into three different prognostic groups for MFS (T1 vs T2: $P=0.039$; T1 vs T3: $P<0.001$; T2 vs T3: $P=0.002$, Fig. S2E). Regarding nodal status, both $\mathrm{N} 1$ and $\mathrm{N} 2$ patients had a significantly worse MFS than node-negative (N0) patients $(P<0.001$ and $P=$ 0.001 , respectively, Fig. S2F). Patients with distant metastases at diagnosis (M1) showed a significantly worse survival than patients without distant metastases at diagnosis (M0) $(P<0.001$, Fig. S2G). Metastasis and death due to breast cancer were defined as events in the metastasis-free survival analyses. All comparisons among the disease stages were significant $(P \leq 0.001)$. Patients with higher stages of disease had a worse MFS than patients with lower stages (Fig. S2H). We used the Mantel-Cox log-rank test to calculate the significance level of differences between OS or MFS distributions for the different LRIG1 copy number categories (loss, normal, or gain) for the whole cohort or early-stage breast cancer (stages I and II), for the entire study period, and for 5 years and 10 years (Fig. 2). The overall survival analysis for all patients demonstrated that patients with LRIG1 gain, but not LRIG1 loss, had a worse prognosis than patients with a normal 


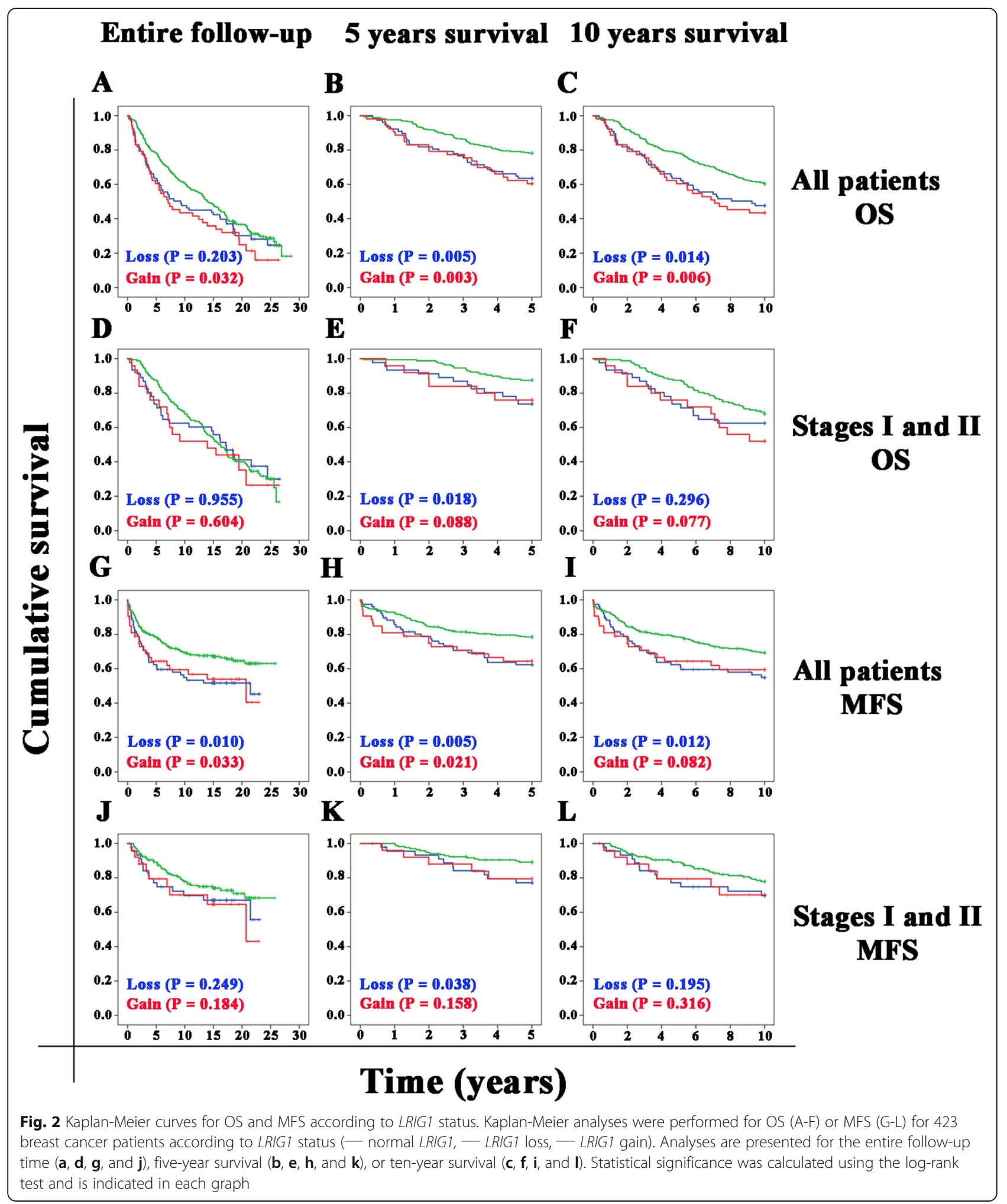

LRIG1 copy number (Fig. 2a). However, for 5-year survival (Fig. 2b) or 10-year survival (Fig. 2c), patients with either LRIG1 loss or LRIG1 gain had a significantly worse OS than patients with a normal LRIG1 copy number. The overall survival analysis for early-stage patients revealed no significant differences between patients with LRIG1 loss or gain and patients with a normal LRIG1 copy number (Fig. 2d). However, for 5-year OS (Fig. 2e), but not for 
10-year OS (Fig. 2f), patients with LRIG1 loss had a significantly worse OS than patients with a normal LRIG1 copy number (Fig. 2e and f). In the entire cohort, both patients with LRIG1 loss and LRIG1 gain had a significantly worse MFS than patients with a normal LRIG1 copy number (Fig. 2g). This pattern was also observed for 5- and 10year MFS (Fig. $2 \mathrm{~h}$ and i). However, for stage I and II patients, only patients with LRIG1 loss in the 5-year MFS analysis showed a significant difference compared with the patients with a normal LRIG1 copy number (Fig. 2j-1). For the early-stage patients who relapsed, the median time to relapse was 43.4 months for patients with LRIG1 loss and 68.5 months for patients with a normal LRIG1 copy number. In our primary Cox regression model (Table 2), we included all the variables that significantly affected OS or MFS in our univariate analyses, i.e., tumor subtype, tumor grade, tumor size, nodal status, and patient age at diagnosis and LRIG1 loss or gain. In this model, tumor subtypes and nodal status were independent prognostic factors both for OS and MFS, whereas tumor size and age at diagnosis were independent prognostic factors for OS only. However, neither LRIG1 loss nor LRIG1 gain showed a significant independent association with patient OS or MFS.
Moreover, we did statistical analyses using the causespecific breast cancer survival estimates together with the metastasis-free survival, but the results were very similar.

\section{Discussion}

The identification of prognostic markers for risk of relapse in breast cancer is of major importance, and loss of LRIG1 has indeed been shown to be a strong candidate marker for the risk of relapse in a stage I-II American breast cancer cohort [16]. To critically evaluate LRIG1 loss as a prognostic marker in other breast cancer cohorts, we devised a precise and robust ddPCR method to assess LRIG1 copy number ratios and applied this method to analyze LRIG1 copy numbers in a healthy control population and a breast cancer cohort from northern Sweden. Among 423 stage I-IV breast cancer cases with a long follow-up period (20 years), we investigated possible associations between LRIG1 copy number and patient survival and various clinical factors. Thereby, we could confirm some and refute other previously published observations regarding LRIG1 copy number associations in breast cancer.

Table 2 Cox regression analysis of LRIG1 loss, normal, and gain adjusted for all variables in all patients

\begin{tabular}{|c|c|c|c|c|}
\hline \multirow{2}{*}{$\begin{array}{l}\text { Tumor } \\
\text { characteristic }\end{array}$} & \multicolumn{2}{|l|}{ Overall survival } & \multicolumn{2}{|c|}{ Metastasis-free survival } \\
\hline & $\begin{array}{l}\text { Hazard ratio } \\
(95 \% \mathrm{Cl})\end{array}$ & $\boldsymbol{P}$-value & $\begin{array}{l}\text { Hazard ratio } \\
(95 \% \mathrm{Cl})\end{array}$ & $\boldsymbol{P}$-value \\
\hline \multicolumn{5}{|l|}{ Age at diagnosis } \\
\hline$\leq 50$ years & Reference & & & \\
\hline$>50$ years & $2.633(1.802-3.848)$ & $<0.001$ & $1.306(0.828-2.060)$ & 0.251 \\
\hline \multicolumn{5}{|l|}{ Tumor subtype } \\
\hline ERBB2-, ER/PR+ & Reference & & & \\
\hline ERBB2+, ER/PR- & $1.558(1.003-2.420)$ & 0.049 & 1.915 (1.077-3.405) & 0.027 \\
\hline ERBB2+, ER/PR+ & $1.563(0.974-2.507)$ & 0.064 & $1.983(1.101-3.573)$ & 0.023 \\
\hline ERBB2-, ER/PR- & 1.384 (0.939-2.038) & 0.1 & $1.583(0.936-2.679)$ & 0.087 \\
\hline \multicolumn{5}{|l|}{ Grade } \\
\hline Low (1 or 2) & Reference & & & \\
\hline High (3) & $1.0(1.0-1.0)$ & 0.710 & $1.583(0.936-2.679)$ & 0.087 \\
\hline \multicolumn{5}{|l|}{ Tumor size } \\
\hline$\leq 20 \mathrm{~mm}$ & Reference & & & \\
\hline$>20 \mathrm{~mm}$ & 1.427 (1.067-1.908) & 0.017 & $1.151(0.751-1.762)$ & 0.519 \\
\hline \multicolumn{5}{|l|}{ Nodal status } \\
\hline Negative & Reference & & & \\
\hline Positive & $2.592(1.840-3.651)$ & $<0.001$ & $3.435(2.206-5.347)$ & $<0.001$ \\
\hline \multicolumn{5}{|c|}{ LRIG1 copy number } \\
\hline Normal & Reference & & & \\
\hline Loss & $0.964(0.668-1.393)$ & 0.847 & $1.115(0.690-1.803)$ & 0.657 \\
\hline Gain & $0.837(0.550-1.274)$ & 0.407 & $1.041(0.585-1.852)$ & 0.892 \\
\hline
\end{tabular}


In accordance with previous studies showing that LRIG1 expression is higher in ER $\alpha$-positive than in ER $\alpha$ negative tumors [12] and decreased in ERBB2-positive compared with ERBB2-negative tumors [13], we found that LRIG1 loss was more common among steroid receptor-negative tumors and ERBB2-positive tumors than among steroid receptor-positive and ERBB2-negative tumors, respectively. In contrast to the results obtained previously [16], however, we found that LRIG1 loss was significantly correlated with tumor grade and nodal status. Thus, in the present study, the frequency of LRIG1 loss seemed to increase together with increasing aggressiveness of the tumor. Moreover, we could confirm that LRIG1 copy numbers were associated with the breast cancer tumor subtype, although the tumor subtype criteria used in the current study, applied to relatively old clinical material, were slightly different from the more modern criteria used by Thompson et al. [16].

A genomic breakpoint has been speculated [18] and shown [16] to be present in LRIG1 in breast cancer; however, the prevalence of this genomic alteration has not been determined previously. Here, we could show that one in 34 breast tumors $(2.9 \%)$ in our cohort appeared to display an unbalanced LRIG1 recombination event. Thus, the frequency of unbalanced LRIG1 recombination events does not seem to be very high in breast cancer, although it will be interesting to analyze larger breast cancer data sets, such as the TCGA data sets, to acquire more reliable estimates of the frequency and to resolve whether specific breast cancer subtypes are predominantly associated with this event.

In the present study, $12.5 \%$ of the tumors displayed LRIG1 gains, contrasting with our previous FISH results $[17,18]$ showing that $39 \%$ of breast tumors displayed LRIG1 gains. In an effort to clarify this discordance, we applied our new ddPCR method to analyze 34 tumors that had previously been analyzed by FISH. The results obtained with ddPCR showed a striking discordance with the previous FISH results. In fact, there was almost no correlation between the results of the two methods. This discordance could not be explained by any difference between the samples analyzed because the ddPCR and FISH analyses were performed on the same material, i.e., the same preparation of cell nuclei from each tumor. In the present ddPCR study, we used a reference gene on another chromosome to normalize the LRIG1 copy number according to the cell number and tumor ploidy, whereas in the previous study, the LRIG1 FISH signals were only normalized to the number of cells, i.e., the number of cell nuclei. It is possible that the lack of agreement between the ddPCR and FISH results might originate from the different normalization strategies used. Hence, we propose that the increased LRIG1 copy numbers previously observed by FISH in most cases may reflect a general polyploidy of the tumor cells rather than specific increases in the LRIG1 gene dosage.

Although the overall prognosis of breast cancer has recently improved [22], many patients still experience recurrence. Therefore, there is a great need for new and reliable tools to predict outcomes and to select the appropriate therapy. Regarding the prognostic value of LRIG1 copy number alterations, both LRIG1 loss and LRIG1 gain were associated with an unfavorable MSF in this study, both for the whole follow-up time and for the 5-year and 10-year survival studies. Thus, LRIG1 status predicted both early and late relapses in our cohort. However, in a multivariate Cox regression analysis, neither LRIG1 loss nor LRIG1 gain was an independent prognostic factor after adjustment for the tumor subtype, tumor grade, LRIG1 copy number status, tumor size, nodal status, and age at diagnosis. Only tumor subtype and nodal status were found to be independent prognostic factors in this analysis. Moreover, among the stage I and II cases, neither LRIG1 loss nor LRIG1 gain was significantly associated with patient survival for the whole study period or the 10-year follow-up. Taken together, these analyses suggest that the observed correlations between LRIG1 status and MFS in the present cohort were probably mostly due to associations between LRIG1 status and tumor subtype and nodal status. These results contrast with our previous demonstration that LRIG1 loss predicts early and late relapses of earlystage breast cancer [16]. The reason for the discordance between our two studies is not known. However, possible explanations include the differences between the patient cohorts analyzed and the analytical methods used. The current cohort comprised 423 patients in total, of whom only 240 were stage I-II, whereas the American cohort comprised 972 patients of stage I-II. In the current cohort, ethnicity was not recorded; however, it is likely that the ethnic compositions of the cohorts were different, which could be highly relevant because black and Hispanic populations are known to have a higher proportion of basal-like and ERBB2-positive tumors than non-Hispanic white populations, and indeed, the frequency of LRIG1 loss differed among these groups in the American cohort [16]. Moreover, another factor with a potential major impact on patient outcome concerns the treatment differences between the cohorts. Regrettably, complete treatment records were not available for the patients in the current study. Another shortcoming of our study was that our clinical material did not comprise mRNA, and therefore LRIG1 mRNA expression analysis could not be performed to clarify its potential role as a prognostic factor in breast cancer. Accordingly, neither was the correlation between LRIG1 gene copy number and LRIG1 expression analyzed in this study. It will be important to further assess these 
associations in larger breast cancer data sets, such as those available from TCGA, the International Cancer Genome Consortium, and the Molecular Taxonomy of Breast Cancer International Consortium.

\section{Conclusions}

By using a novel ddPCR-based LRIG1 copy number assay, we have shown that LRIG1 loss is associated with nodal status and other clinical parameters; however, we could not verify LRIG1 loss as a robust independent predictor of the risk of relapse in breast cancer. Thus, LRIG1 gene aberrations may be important biological determinants of various aspects of breast cancer biology, and considered as prognostic markers, but the role of this gene as an independent predictor of relapse in breast cancer appears uncertain.

\section{Supplementary information}

Supplementary information accompanies this paper at https://doi.org/10. 1186/s12885-020-06919-w.

Additional file 1: Fig. S1. Lack of a correlation between the $d d P C R$ and FISH results. Dot plot showing LRIG1 copy numbers determined for 34 breast tumors using ddPCR (current study) and using FISH (Ljuslinder et al., 2009). The linear regression line $\left(y=1.004+0.100 x, r^{2}=0.009\right)$ is presented as a broken blue line.

Additional file 2: Fig. S2. Kaplan-Meier curves for MFS according to known risk factors. Kaplan-Meier analyses were performed for 423 breast cancer patients according to ER status (A), ERBB2 status (B), breast cancer subtype $(C)$, tumor grade $(D)$, tumor size $(E)$, nodal status $(F)$, distant metastasis $(\mathrm{G})$, and disease stage $(\mathrm{H})$. Statistical significance was calculated using the log-rank test and is indicated in each graph.

Additional file 3.

\section{Abbreviations}

Cl: Confidence interval; CV: Coefficient of variation; ddPCR: Droplet digital PCR; ER: Estrogen receptor; FISH: Fluorescence in situ hybridization; LRIG1: Leucine-rich repeats and immunoglobulin-like domains 1; MFS: Metastasis-free survival; OS: Overall survival; PCR: Polymerase chain reaction; PR: Progesterone receptor; SD: Standard deviation; TCGA: The cancer genome atlas

\section{Acknowledgments}

\section{Not applicable.}

\section{Authors' contributions}

MF performed experiments, interpreted results, and wrote the manuscript. AT performed experiments and interpreted results. CEA analyzed patient records. KG performed sample procurement and interpreted results. LH performed the bioinformatics analyses of copy number variations in the TCGA data sets. BT performed statistical analyses and interpreted results. $\mathrm{RH}$ raised funding, performed sample procurement, and interpreted results. IL raised funding, performed sample procurement, designed the study, interpreted results, and wrote the manuscript. $\mathrm{HH}$ raised funding, designed the study, interpreted results, and wrote the manuscript. All authors read and approved the final manuscript.

\section{Funding}

This work was supported by grants from the Swedish Cancer Society, the Cancer Research Foundation in Northern Sweden, and by the regional agreement between Umeå University and Västerbotten County Council on the cooperation in the field of Medicine, Odontology and Health (ALF). The funding bodies had no role in the design of the study or collection, analysis, or interpretation of data or in writing the manuscript.
Open access funding provided by Umea University.

\section{Availability of data and materials}

The data sets are available from the corresponding author on reasonable request.

\section{Ethics approval and consent to participate}

The study protocol and data handling procedures were approved by the Regional ethical review board at Umeå University, Umeå, Sweden (DNR 02 455). As the cohort is more than 20-30 years old and most of the patients have passed away, the mentioned ethics committee approved the study without consent from the patients or their relatives.

\section{Consent for publication \\ Not applicable.}

\section{Competing interests}

The authors declare that they have no competing interests.

\section{Author details}

'Department of Radiation Sciences, Oncology, Umeå University, SE-90187 Umeå, Sweden. ${ }^{2}$ Department of Medical Biosciences, Umeå University, SE-90187 Umeå, Sweden. ${ }^{3}$ National Bioinformatics Infrastructure Sweden, SciLifeLab, Uppsala, Sweden. ${ }^{4}$ Current address: Instytut Genetyki i Hodowli Zwierząt Polskiej Akademii Nauk, ul. Postępu 36A, 05-552 Jastrzębiec, Magdalenka, Poland.

Received: 13 December 2019 Accepted: 30 April 2020

Published online: 24 May 2020

\section{References}

1. Ferlay J, Shin HR, Bray F, Forman D, Mathers C, Parkin DM. Estimates of worldwide burden of cancer in 2008: GLOBOCAN 2008. Int J Cancer. 2010; 127(12):2893-917.

2. DeSantis C, Ma J, Bryan L, Jemal A. Breast cancer statistics, 2013. CA Cancer J Clin. 2014;64(1):52-62.

3. Perou CM, Sorlie T, Eisen MB, van de Rijn M, Jeffrey SS, Rees CA, Pollack JR, Ross DT, Johnsen H, Akslen LA, et al. Molecular portraits of human breast tumours. Nature. 2000;406(6797):747-52

4. Sorlie T, Perou CM, Tibshirani R, Aas T, Geisler S, Johnsen H, Hastie T, Eisen $M B$, van de Rijn M, Jeffrey SS, et al. Gene expression patterns of breast carcinomas distinguish tumor subclasses with clinical implications. Proc Natl Acad Sci U S A. 2001;98(19):10869-74.

5. Lehmann BD, Bauer JA, Chen X, Sanders ME, Chakravarthy AB, Shyr $Y$, Pietenpol JA. Identification of human triple-negative breast cancer subtypes and preclinical models for selection of targeted therapies. J Clin Invest. 2011;121(7):2750-67.

6. Lowery AJ, Kell MR, Glynn RW, Kerin MJ, Sweeney KJ. Locoregional recurrence after breast cancer surgery: a systematic review by receptor phenotype. Breast Cancer Res Treat. 2012;133(3):831-41.

7. Voogd AC, Nielsen M, Peterse JL, Blichert-Toft M, Bartelink H, Overgaard M, van Tienhoven G, Andersen KW, Sylvester RJ, van Dongen JA, et al. Differences in risk factors for local and distant recurrence after breastconserving therapy or mastectomy for stage I and II breast cancer: pooled results of two large European randomized trials. J Clin Oncol Off J Am Soc Clin Oncol. 2001;19(6):1688-97.

8. Soerjomataram I, Louwman MW, Ribot JG, Roukema JA, Coebergh JW. An overview of prognostic factors for long-term survivors of breast cancer. Breast Cancer Res Treat. 2008;107(3):309-30.

9. Oven Ustaalioglu BB, Balvan O, Bilici A, Develi A, Aliustaoglu M, Vardar FA, Erkol B. The differences of clinicopathological factors for breast cancer in respect to time of recurrence and effect on recurrence-free survival. Clin Transl Oncol. 2015;17(11):895-902.

10. Gur G, Rubin C, Katz M, Amit I, Citri A, Nilsson J, Amariglio N, Henriksson R, Rechavi G, Hedman H, et al. LRIG1 restricts growth factor signaling by enhancing receptor ubiquitylation and degradation. EMBO J. 2004;23(16): 3270-81.

11. Laederich MB, Funes-Duran M, Yen L, Ingalla E, Wu X, Carraway KL 3rd, Sweeney $C$. The leucine-rich repeat protein $L R I G 1$ is a negative regulator of ErbB family receptor tyrosine kinases. J Biol Chem. 2004;279(45):47050-6. 
12. Krig SR, Frietze S, Simion C, Miller JK, Fry WH, Rafidi H, Kotelawala L, Qi L, Griffith OL, Gray JW, et al. Lrig1 is an estrogen-regulated growth suppressor and correlates with longer relapse-free survival in ER alpha-positive breast cancer. Mol Cancer Res. 2011;9(10):1406-17.

13. Miller JK, Shattuck DL, Ingalla EQ, Yen L, Borowsky AD, Young L, Cardiff RD, Carraway KL 3rd, Sweeney C. Suppression of the negative regulator LRIG1 contributes to ErbB2 overexpression in breast cancer. Cancer Res. 2008; 68(20):8286-94.

14. Yokdang N, Hatakeyama J, Wald JH, Simion C, Tellez JD, Chang DZ, Swamynathan MM, Chen M, Murphy WJ, Carraway lii KL, et al. LRIG1 opposes epithelial-to-mesenchymal transition and inhibits invasion of basallike breast cancer cells. Oncogene. 2016;35(22):2932-47.

15. Lindquist D, Kvarnbrink S, Henriksson R, Hedman H. LRIG and cancer prognosis. Acta Oncol. 2014;53(9):1135-42.

16. Thompson PA, Ljuslinder I, Tsavachidis S, Brewster A, Sahin A, Hedman H, Henriksson R, Bondy ML, Melin BS. Loss of LRIG1 locus increases risk of early and late relapse of stage I/II breast cancer. Cancer Res. 2014;74(11):2928-35.

17. Ljuslinder I, Malmer B, Golovleva I, Thomasson M, Grankvist K, Hockenstrom T, Emdin S, Jonsson Y, Hedman H, Henriksson R. Increased copy number at 3p14 in breast cancer. Breast Cancer Res. 2005;7(5):R719-27.

18. Ljuslinder I, Golovleva I, Henriksson R, Grankvist K, Malmer B, Hedman H. Coincidental increase in gene copy number of ERBB2 and LRIG1 in breast cancer. Breast Cancer Res. 2009;11(3):403.

19. Linderholm BK, Lindh B, Beckman L, Erlanson M, Edin K, Travelin B, Bergh J, Grankvist K, Henriksson R. Prognostic correlation of basic fibroblast growth factor and vascular endothelial growth factor in 1307 primary breast cancers. Clin Breast Cancer. 2003;4(5):340-7.

20. Linderholm B, Lindh B, Tavelin B, Grankvist K, Henriksson R. p53 and vascular-endothelial-growth-factor (VEGF) expression predicts outcome in 833 patients with primary breast carcinoma. Int J Cancer. 2000;89(1):51-62.

21. Thompson PA, Brewster AM, Kim-Anh D, Baladandayuthapani V, Broom BM, Edgerton ME, Hahn KM, Murray JL, Sahin A, Tsavachidis S, et al. Selective genomic copy number imbalances and probability of recurrence in earlystage breast cancer. PLoS One. 2011;6(8):e23543.

22. Miller KD, Siegel RL, Lin CC, Mariotto AB, Kramer JL, Rowland JH, Stein KD, Alteri R, Jemal A. Cancer treatment and survivorship statistics, 2016. CA Cancer J Clin. 2016:66(4):271-89.

\section{Publisher's Note}

Springer Nature remains neutral with regard to jurisdictional claims in published maps and institutional affiliations.

Ready to submit your research? Choose BMC and benefit from:

- fast, convenient online submission

- thorough peer review by experienced researchers in your field

- rapid publication on acceptance

- support for research data, including large and complex data types

- gold Open Access which fosters wider collaboration and increased citations

- maximum visibility for your research: over $100 \mathrm{M}$ website views per year

At $\mathrm{BMC}$, research is always in progress.

Learn more biomedcentral.com/submissions 